\title{
(1D Comparative analysis of memory impairment after general anesthesia in different age groups
}

\section{8 \\ E. Sinbukhova1 , A. Lubnin¹, G. Danilov², N. Konovalov³}

1Department of Anaesthesiology and Intensive care, ${ }^{2}$ Academic Secretary, ${ }^{3}$ Spinal neurosurgical Department

“N. N. Burdenko National Medical Research Center of Neurosurgery" of Ministry of Health of the Russia Federation

Abstract: Various studies indicated that general anesthesia led to a significant risk of early postoperative cognitive dysfunction (POCD) in elderly patients. The incidence of POCD is often underestimated, it usually occurs in elderly patients but memory problems of younger patients undergoing general anesthesia have not been studied thoroughly.

Hypothesis: The application of general anesthesia leads to memory impairment in young and middle aged patients.

Objective: To assess memory decline in different age groups of patients after the spinal neurosurgical treatment with general anesthesia (propofol and fentanyl).

Methods: Neurocognitive tests according to Luria, 10 words test, visuospatial memory testing (complex images), Wechsler Memory Scale (WAIS) - Russian adaptation, Montreal Cognitive Assessment (MoCA), HADS, Pain Detect were used. Neurocognitive tests were performed preoperatively and at the moment of discharge.

Keywords: cognitive dysfunction, memory impairment, anesthesia.

The data have been prepared in Microsoft Excel 2011. The statistical analysis was performed in R-Studio (www.r-project.org). The methods of descriptive statistics were used, hypothesis testing on the differences in groups were conducted using Mann-Whitney $U$ test.

Results: In our study 60 patients with chronic back pain undergoing neurosurgical treatment. Anesthesia duration was 4 hours on average. The underlying pathology was spondylolisthesis, degenerative stenosis, hernia, tumors.

The inclusion criteria: patients with chronic back pain prepared for elective surgery, age 18-60 years.

The exclusion criteria: MoCA $<16, \mathrm{FAB}<12$ points, kidney and liver failure, chronic heart failure, adrenal insufficiency, diabetes, hormonal disorders, mental illness, chronic alcohol or drug abuse, history of brain tumor or traumatic brain injury, previous surgery under general anesthesia.

Patients were divided into two equal groups of 30 persons in each: Group A consisted of young patients (avg. age 32.37 (range 18-44), Group $B$ included middle aged patients (avg. age 51.33 (range 45-60).

Neurocognitive tests were performed preoperatively and at the moment of discharge.

Neurocognitive tests according to Luria: cognitive behavior, $\underline{\mathrm{O}}$ attention, memory, learning verbal function and academic skills, visual perception and visual reasoning, construction, concept formation, executive function, self-regulation, motor ability, emotional ह status - showed a significant decrease in 6 days after general anesthesia in both groups.

The results for group $A$ : ( $n=30$, median \pm standard deviation): 10 words test (deferred naming): before surgery median $=6,5$ $( \pm 1,3)$, at the moment of discharge $=4 \quad( \pm 0,8), p=1.371 \mathrm{e}-09$; Visuospatial memory testing (complex of 5 images): before surgery median $=4( \pm 0,7)$, at the moment of discharge $=2( \pm 0,9), p=4.75 e-$ 11; MoCA: before surgery median $=26(+2,4)$, at the moment of discharge $=25(+1,6), p=0.0007843$; HADS anxiety level: before surgery median $=16( \pm 2,2)$, at the moment of discharge $=14( \pm 2,5)$, $p=0.0003992$; HADS depression level: before surgery median $=12$ $( \pm 4)$, at the moment of discharge $=11( \pm 3,3), p=0.09886$; Pain Level (on average): before surgery median $=9(+2,1)$, at the moment of discharge $=2( \pm 1), p=1.31 \mathrm{e}-10$; WAIS before surgery and at the moment of discharge: 18-19 age: $p=0.3333,20-24$ age: $p$ $=0.002165,25-34$ age: $p=0.002022,35-44$ age: $p=5.276 \mathrm{e}-06$.

The results for group $B:(n=30$, median \pm standard deviation):

10 words test (deferred naming): before surgery median $=7(+1,3)$, at the moment of discharge $=4( \pm 0,8), p=1.075 e-09$; Visuospatial memory testing (complex of 5 images): before surgery median $=3$ $( \pm 1)$, at the moment of discharge $=1( \pm 0,8), p=2.322 e-09$;
MoCA: before surgery median $=26( \pm 2,6)$, at the moment of discharge $=25( \pm 1,8), p=0.004485$; HADS anxiety level: before surgery median $=$ $16( \pm 3)$, at the moment of discharge $=14( \pm 2,7), p=0.000596$;

HADS depression level: before surgery median $=12( \pm 4)$, at the moment of discharge $=10,5 \quad( \pm 3,7), p=0.02662$; Pain Detect - pain Level (on average): before surgery median $=9( \pm 2,3)$, at the moment of discharge $=2( \pm 1,6), p=6.818 e-10$;WAIS before surgery and at the moment of discharge: $45-54$ age: $p=8.735 e-08,55-60$ age: $p=$ 0.0002906

Comparison of groups $A$ and $B$ at the moment of discharge:

- 10 words test (deferred naming): $p=0.622$

- Visuospatial memory testing (complex of 5 images): $p=0.3651$

- MoCA: $p=0.9879$

- HADS anxiety level: $p=1$

- HADS depression level: $p=0.7145$

- Pain Level: $p=0.4728$

- Wechsler Memory Scale (18-44 and 45-60 age): $p=0.3826$

30

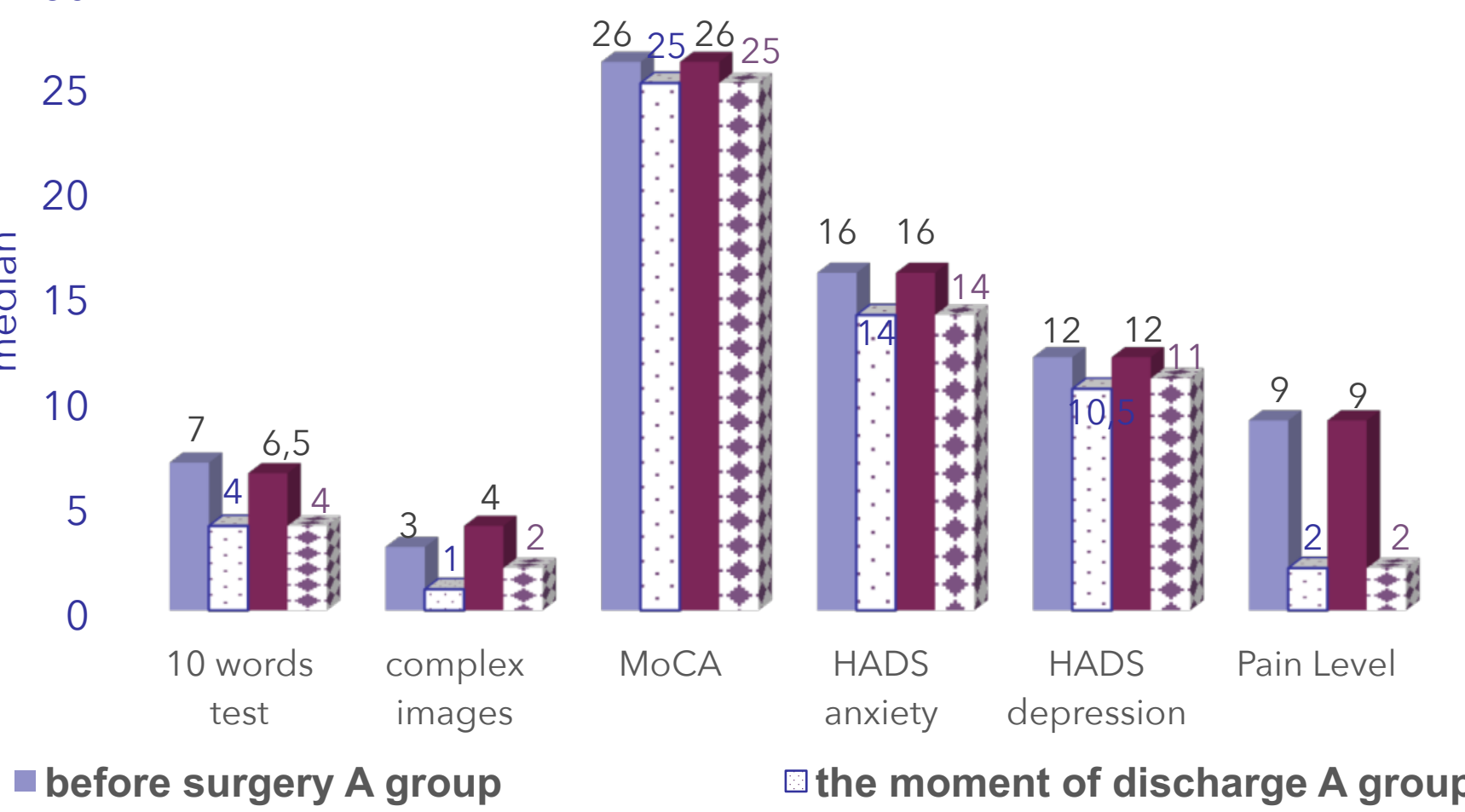

- before surgery B group

: the moment of discharge B group

Fig.1. Patients in Groups A and B: before surgery and at the moment of discharge: 10 words test, Visuospatial memory testing (complex images), MoCA, HADS anxiety level, HADS depression level, Pain Level. (Median).

150

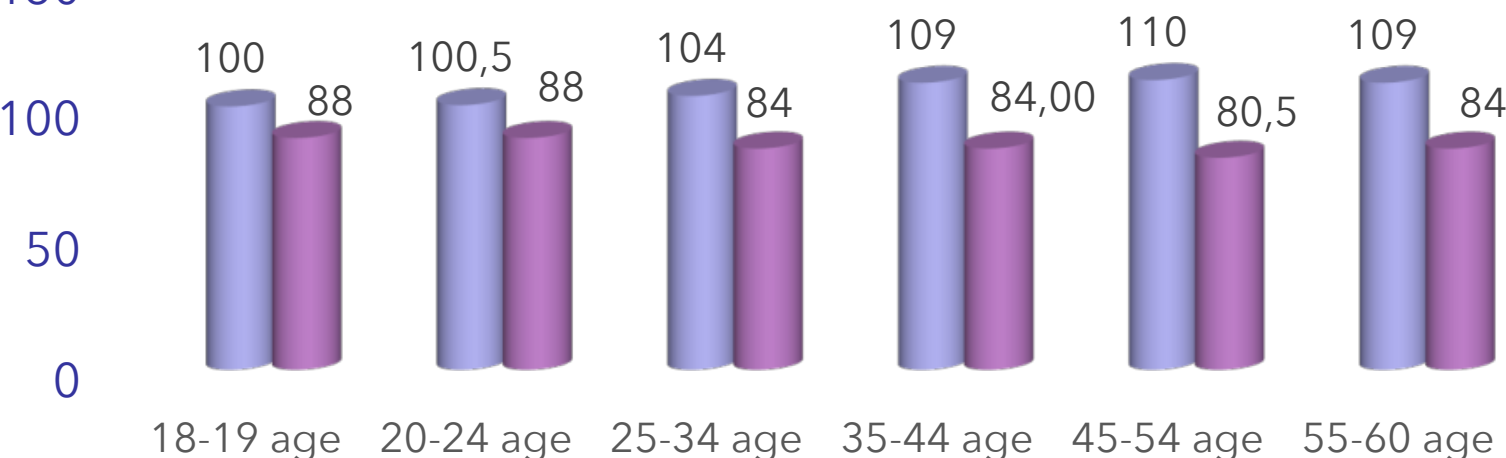

-before surgery $\quad$ the moment of discharge

Fig. 2. Patients in Group A and B: before surgery and at the moment of discharge (according to age): Wechsler Memory Scale (Median).

- As we could see at Fig. 2. before surgery median meaning is medium - IQ all ages, at the moment of discharge IQ median meaning is bad norm

\section{Conclusions}

Results of our study showed a significant memory decrease in 6 days after general anesthesia. A significant memory decrease was observed in both groups 6 days after anesthesia.

Thus it is necessary to conduct further studies of possible postoperative cognitive dysfunction and ways of its prevention and correction in younger patients and middle age patients after using general anesthesia during surgery treatment, including patients with chronic back pain. 